
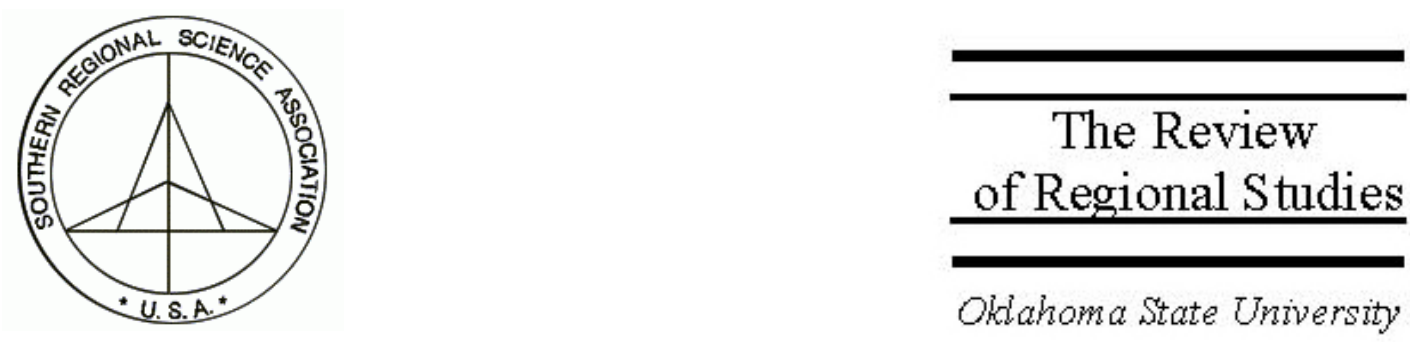

\title{
Sensitivity of CGE Simulation Results to Competing SAM Updates
}

\author{
M. Alejandro Cardenete \\ Departamento de Economía y Empresa and CentrA, Universidad Pablo de Olavide de \\ Sevilla, Carretera de Utrera s/n, km. 1, 41013-Sevilla, Spain, \\ email: macarflo@dee.upo.es \\ Ferran Sancho \\ Departament d'Economia and CREA, Universitat Autònoma de Barcelona, Campus de \\ Bellaterra,08193-Bellaterra, Spain, email: ferran.sancho@uab.es
}

\begin{abstract}
Recent research shows a renewed interest in the properties of non-survey updates of input-output tables and social accounting matrices (SAM). Along with the scaling RAS method, new procedures related to entropy minimization and other metrics have been examined. Whether they will replace or complement the RAS method is an open question. The performance of the updating procedures has been tested with proximity measures to a reference input-output table or SAM, but we look at the performance of three updating mechanisms by comparing the simulation results from adopting alternative databases for the calibration of a reference CGE model.
\end{abstract}

Keywords: Social accounting matrices; Input-output; Non-survey updating techniques; Computable general equilibrium; Regional policy analysis; Evaluation of simulation results

JEL Classification: C52; C67; C68

The second author thanks Catalonia's Department of Universities, Research and Information Society (DURSI) Mobility Programme BE2002-400105 for support to visit the University of California at Berkeley, where this paper was developed. The research is part of ongoing projects SEC2003-5112 from Spain's Ministry of Science and Technology and SGR2001-0164 from DURSI. We thank participants at the 2003 Annual North American meetings of the Regional Science Association International, where a version was presented, two referees, and Dan Rickman for helpful suggestions. Errors and shortcomings remain the authors' responsibility. 


\section{INTRODUCTION}

Most often the data in a social accounting matrix (SAM) are used to implement empirical multisectoral and computable general equilibrium (CGE) models, which are in turn used to perform economic analyses and policy simulations. The quality of data and/or its timeliness are therefore of critical relevance in appraising and evaluating model results and to endowing them with increased credibility before policy makers. Unfortunately, data of the kind needed in multisectoral modeling are not produced in a timely and regular way by statistical offices. Data collection and compilation is expensive, and an excessive temporal lag in the production and publication of official data is often an unavoidable reality. Indirect ways out of this problem do exist in the form of updating techniques that permit the projection of a base year SAM. ${ }^{1}$ In fact, the SAM updating problem is nothing but a particular case of the well-known matrix-balancing problem of the linear algebra literature (Rothblum and Schneider 1989; Schneider and Zenios 1990). The technique most commonly used in updating a SAM is the RAS or biproportional method. The appeal of RAS arises from its extremely simple algorithmic implementation and its applicability (Jensen 1980). Its conceptual and mathematical properties are fully described in Bacharach (1970). More recently, entropy techniques from information theory have been adapted by Golan, Judge, and Robinson (1994); Thissen and Logfren (1999); and Robinson, Cattaneo, and El-Said (2001) for the updating of input-output tables and social accounting matrices. However RAS and entropy methods are closely related, as Bacharach (1970, chapter 6), Schneider (1989), Schneider and Zenios (1990), and McDougall (1999) have pointed out. Indeed, in addition to the iterative scaling method of rows and columns, RAS can also be formulated as a nonlinear entropy minimization problem for the matrix of total transactions.

Accepting that SAM data are not readily available in as regular a basis as desired, updating techniques that use prior data plus partial new information will somehow alleviate the problem of not having an actual newer SAM. ${ }^{2}$ If we denote by $A^{0}$ an available SAM matrix, an updated matrix $\hat{A}^{1}$ is a projection of the matrix $A^{0}$; but it is also an estimate of the "true" unknown matrix $A^{1}$. The distance between $A_{0}$ and $\hat{A}^{1}$, however minimized, entails an error, unknown in magnitude if $A^{1}$ is itself unknown, between $\hat{A}^{1}$ and the true matrix $A^{1}$, as Jian (2002) has recently shown using Monte Carlo simulations. When the true matrix is finally available, it is possible to measure ex-post the accuracy involved in each of the different updating procedures. Limited to input-output tables, this is the approach followed by Jensen (1980), Szyrmer (1989), and Jackson and Murray

\footnotetext{
${ }^{1}$ An input-output table can always be embedded in a SAM as a data subset. When no need for clarification is required, we will refer to SAM updating as a term encompassing both a SAM proper and an input-output table.

${ }^{2}$ An additional advantage of building an updated SAM is that it can be used for non-CGE multisectoral modeling. If a CGE model is all that matters and sufficient data exists, then Arndt, Robinson, and Tarp (2002) provide an alternative approach to parameter specification.
} 
(2003), who present a thorough discussion and testing of the RAS procedure in terms of ex-post accuracy and prediction power. But when the true matrices are unknown, the usual recourse is to perform an ex-ante evaluation measuring the proximity between the given initial matrix and the updated ones (see Thissen and Logfren 1999 and Robinson, Cattaneo, and El-Said. 2001).

We wish to call the attention, to the fact that measuring the ex-ante distance performance between the base matrix $A^{0}$ and its possible updatings $\hat{A}^{1}$, and the ex-post error between $A^{1}$ and $\hat{A}^{1}$ whenever this becomes feasible, is clearly necessary. We feel, however, that is not entirely sufficient. Distance and error appraisals can and should be complemented with an ex-ante analysis of the variability induced in multisectoral and CGE simulation results by the adoption of updated databases in place of the true unavailable one. After all, the main purpose of updating a database is to use it to calibrate and solve more current or up-to-date models that can then be used to perform policy analyses. To our knowledge, the literature thus far has not explored the implications of different updating techniques for the CGE simulation results that alternative calibrations will produce.

As a first step in this direction, we consider three different updating procedures to project forward in time a 1995 regional SAM of Andalusia, Spain, to known total marginals for 1999. Using RAS, cross-entropy (CE), and a new procedure based on information retrieval principles, we generate three competing microconsistent SAMs. The choice of these techniques is justified by their informational content. Many other procedures based on different metrics exist, as Jackson and Murray (2003) show, but standard vector metrics sometimes lack a clear-cut informational or economic interpretation.

Once we obtain the three competing SAMs, we calibrate a CGE regional tax model developed by the authors (see Cardenete and Sancho 2003) and proceed to perform a range of policy simulations under the three calibrated versions. In Section 2 we succinctly present the updating techniques, a summary description of the underlying CGE model, and the policy comparisons. Section 3 contains the numerical results and a discussion. Section 4 concludes the paper.

\section{METHODOLOGY}

\subsection{The Matrix Balancing Problem in an Economic Setting}

Let $A=\left(a_{i j}\right)$ represent a matrix in the set $\mathcal{A}_{n}$ of the $n \times n$ non-negative matrices that have no zero row or column. Consider now a matrix $A^{0} \in \mathcal{A}_{n}$, a positive vector $X \in R_{+}^{n}$, and a loss function $d: \mathcal{A}_{n} \times \mathcal{A}_{n} \rightarrow R$. The matrix-balancing problem consists in finding a 
new matrix $\hat{A}^{1} \in \mathcal{A}_{n}$ that solves the following programming problem. ${ }^{3}$

$\operatorname{Min} d\left(A^{0}, \hat{A}^{1}\right)$

subject to

(i) $\sum_{j=1}^{n} \hat{a}_{i j}^{1}=X_{i}$ for all $i$

(ii) $\sum_{i=1}^{n} \hat{a}_{j i}^{1}=X_{j}$ for all $j$

(iii) $a_{i j}^{0}=0$ implies $\hat{a}_{i j}^{1}=0$

The given matrix $A^{0}$ incorporates the structural "prior” information that is known; and that along with the "new" partial information in $X$ will be used to produce the solution, or "projected”, matrix $\hat{A}^{1}$. Mathematically, restrictions (i) and (ii) establish that both the column and the row sums of the solution matrix $\hat{A}^{1}$ must coincide with the values set in the new marginal vector $X$. Restriction (iii) makes the updated matrix inherit the zero structure of the base matrix. ${ }^{4}$

In an economic setting, matrices $A^{0}$ and $\hat{A}^{1}$ would represent socioeconomic SAMs, whereas the vector $X$ would incorporate updated information on row and column marginal totals. The most typical situation is the projection of a SAM at date $t=0$ to a later date $t=1$ for which new marginal totals are known. A related problem is the regionalization of a national SAM where the vector $X$ corresponds to regional level data. In this case, $A^{0}$ would be a national SAM and $\hat{A}^{1}$ the adjusted regional SAM. The analytical structure of the problem is nonetheless the same.

It is the nature of the function $d$ that gives rise to alternate updating results. For instance, in the RAS procedure we seek a projected SAM $\hat{A}^{1}$ through the minimization of:

$$
d\left(A^{0}, \hat{A}^{1}\right)=\sum_{i=1}^{n} \sum_{j=1}^{n} \hat{a}_{i j}^{1} \cdot\left(\ln \hat{a}_{i j}^{1}-\ln a_{i j}^{0}\right)
$$

\footnotetext{
${ }^{3}$ In the general presentation of the matrix balancing problem, new marginal vectors for row and column sums need not be identical. For a SAM matrix, however, they necessarily coincide because of the budget constraint restrictions for all agents and institutions.

${ }^{4}$ There are two types of zero entries in a SAM. The first type are "technical" zeros; this is the case of an input that is not actually used in the production of a commodity but could be used under a different technology. The second type are "conceptual" zeros. In no SAM is labor directly used in the production of consumption nor in generating excise taxes. The updating cannot be allowed to change these second type of zero entries into non-zero entries. We believe the safest course of action is to maintain the zero structure of the prior matrix
} 
subject to restrictions (i) to (iii). As is well known, the solution of this programming problem yields the same result as the biproportional-scaling algorithm (Bacharach 1970; McDougall 1999; Jackson and Murray 2003). Although the computational requirements for the programming approach are heavier than for the scaling method, the problem is feasible with today's powerful computing software. On the other hand, a clear advantage of the programming technique is that if additional partial information related to some of the matrix entries should happen to be available, its incorporation into the minimization program is quite straightforward (Robinson, Cattaneo, and El-Said 2001).

The cross-entropy (CE) approach involves projecting technical coefficients instead of total SAM flows. Once the new coefficients have been obtained, the new SAM can be derived in the usual way. Because cross entropy aims directly at estimating technical coefficients, the scaling method does not work. The difference therefore with regard to standard RAS is that CE uses technical coefficient matrices in the minimand instead of total flows. The program would now consist of minimization:

$$
d\left(A^{0}, \hat{A}^{1}\right)=\sum_{i=1}^{n} \sum_{j=1}^{n}\left(\hat{a}_{i j}^{1} / X_{j}\right) \cdot\left(\ln \left(\hat{a}_{i j}^{1} / X_{j}\right)-\ln \left(a_{i j}^{0} / X_{j}^{0}\right)\right)
$$

subject again to (i) to (iii). Here $X_{j}^{0}=\sum_{i} a_{i j}^{0}$ is the level value for the $j$ th row and column sum in the original matrix, and $a_{i j}^{0} / X_{j}^{0}$ and $\hat{a}_{i j}^{0} / X_{j}$ initial and updated technical coefficients, respectively. Observe that the RAS and CE programs differ only in the variables they try to estimate (SAM flows and SAM coefficients, respectively) but that they share the same information theory background. RAS, however, can also be implemented using the scaling algorithm.

Many other distances from metric spaces besides the RAS and CE minimands are available to minimize the loss function, but either they do not seem to outperform RAS or somehow their interpretation in terms of information theory or economic content is not immediate (see Jackson and Murray 2003). A possible complement to RAS and CE is suggested by classical information retrieval theory, a branch of computer science concerned with developing efficient methods of retrieving information from a data bank (Salton and McGill 1983). Whenever a query for data is formulated, a retrieval algorithm fetches documents in a data bank that are in some sense closely related to the query. The closer the similarity between the query and the information contained in the retrieved documents, the more successful is the algorithm. Notice that a base SAM can be seen as a query for the true but unknown document SAM, and an information retrieval algorithm will fetch from the data bank (the set of feasible SAMs) one with information content closely matching that required by the query.

The performance of a retrieval algorithm in vector space can be evaluated using similarity indices that measure the degree of proximity, or match, between a query and a retrieved document. Queries and documents are commonly represented by on/off binary values that describe the objects' characteristics, but proximity can be extended in a straightforward manner to continuous values. There are in fact a variety of similarity 
measures; but because of its very simple mathematical structure and its easy economic interpretation, we will focus exclusively here on the cosine similarity index. Consider any two non-negative, non-zero vectors $x, y \in R_{+}^{n}$, their inner product $\langle x, y\rangle$, and their angle $\theta(x, y)$ in Euclidean space. From elementary geometry we know:

$$
\langle x, y\rangle=\cos \theta(x, y) \cdot\|x\| \cdot\|y\|
$$

where $\|x\|$ is the Euclidean norm of vector $x$ and cos is the cosine function. We now argue that the cosine of angle $\theta(x, y)$ can be interpreted as a similarity between $x$ and $y$. Rewriting (3) as:

$$
\cos \theta(x, y)=\frac{\langle x, y\rangle}{\|x\| \cdot\|y\|}
$$

we see that the following properties hold.

a) $0 \leq \cos \theta(x, y) \leq 1$ for all non-zero vectors $x, y \in R_{+}^{n}$ (bounds for similarity).

b) If $x=y$, then $\cos \theta(x, y)=1$ (maximum similarity).

c) If $x$ and $y$ are orthogonal, that is $\langle x, y\rangle=0$, then $\cos \theta(x, y)=0$ (maximum dissimilarity).

Property a) sets natural lower and upper bounds for the vector relationship and follows from the trigonometric definition of cosine. Property b) establishes that like vectors have maximum similarity, whereas c) states that orthogonal vectors have zero similarity. As a matter of fact, property c) has a nice economic interpretation. If $x$ and $y$ are two input requirement vectors for some given level of output, orthogonality means that the two technologies do not share any specific inputs $\left(x_{i}>0\right.$ implies that $y_{i}=0$ and, vice versa, $y_{i}>0$ implies $\left.x_{i}=0\right)$. It is all but natural that the technological similarity should be zero since the input requirement vectors are as technologically far apart as possible in vector space, and this is correctly captured by the cosine measure. The closer two vectors are, the smaller the angle they conform and the larger their similarity is.

Cosine similarity yields a proximity measure that can be used to solve the matrixbalancing problem. Given a base matrix $A^{0}$ and a retrievable matrix $\hat{A}^{1}$, we define for each pair of columns in position $j$ their angle $\theta_{j}\left(a_{i j}^{0}, \hat{a}_{i j}^{1}\right)$. Notice that $\cos \theta_{j}\left(a_{i j}^{0}, \hat{a}_{i j}^{1}\right)$ is a column-wise measure of the technological (or cost structure, or activities) similarity between the two SAM columns in the same position $j$. Adding-up all column similarities to obtain a global similarity index, we can define a loss function (interpreted in this case as dissimilarity) by: 


$$
d\left(A^{0}, \hat{A}^{1}\right)=-\sum_{j=1}^{n} \cos \theta\left(a_{i j}^{0}, \hat{a}_{i j}^{1}\right),
$$

which can then be used as the minimand in the matrix-balancing problem ${ }^{5}$. The solution of the nonlinear programming problem is thus equivalent to retrieve from $A_{n}$ a feasible matrix as cosine similar as possible to the base matrix. And since cosine similarity can be interpreted as technological proximity, the minimization problem has a clear economic interpretation: the retrieved matrix, conditional on the restrictions imposed by the new partial information on $X$, will be most similar in cost structure to the base one.

\subsection{The Regional CGE Model}

The CGE model represents the economy of the Spanish region of Andalusia in 1995, the most recent year for which a regional SAM could be assembled using official data. The model, described in Cardenete and Sancho (2003), is a tax policy model that follows the seminal Shoven-Whalley (1984) tradition. A brief description follows. All markets are competitive and technological, and behavioral functions are linearly homogeneous. Production takes place under a nesting structure combining simple Cobb-Douglas and Leontief production functions. Factors are assumed to be mobile among sectors; but labor can be underused in equilibrium, giving rise to involuntary unemployment. This is accomplished in the model with a feedback function between the real wage and the unemployment rate that is related to the degree of labor market flexibility. Consumers maximize a Cobb-Douglas utility aggregator over present and future consumption under a net income budget constraint. Net income is gross factors income adjusted by an income tax and a collection of transfers from the government and abroad. Final prices for goods, services, and factors are inclusive of indirect taxes (value-added, output, and payroll taxes).

The government collects taxes from consumers and firms and in exchange provides social transfers to consumers and subsidies to firms, purchases public consumption, and undertakes public investment projects. Tax collection is governed by an endogenous tax revenue function that depends on prices and activity levels. The equilibrium concept corresponds closely to the standard Arrow-Debreu concept for linearly homogeneous technologies along with an additional tax revenue equilibrium condition (Ballard et al. 1985) and an adapted labor market equilibrium condition. This general type of model's existence follows from the standard theorems, whereas uniqueness has been tested by Kehoe and Whalley (1985). Meaningful comparative static exercises can therefore be undertaken since the equilibrium set will vary smoothly and without jumps with the exogenous tax structure.

${ }^{5}$ Another widely used measure of similarity is Jaccard similarity (Salton and McGill, 1983). The Jaccard index in vector space is defined as $\operatorname{Jac}(x, y)=\langle x, y\rangle /\left(\sum x_{i}^{2}+\sum y_{i}^{2}-\langle x, y\rangle\right)$. It satisfies the same mathematical properties as cosine similarity, the difference being the normalization. This measure is commonly used in the biological sciences to compare populations. 


\subsection{Updating Strategies and Policy Controls}

Let us assume there is an economy-wide policy reform to be implemented in period $t=1$ (or region $r=1$ ) for which a CGE model is deemed the most adequate analytical tool. Under first-best conditions of full data availability, the CGE model would be calibrated using the latest available true SAM $A^{1}$. In many practical situations, however, such a SAM will not exist; the only usable database will be an older, or locationally different, $A^{0}$ SAM and a vector $X$ of new marginals. In this second-best world, two options are open to the modeler.

1. Calibrate the CGE model to the base $A^{0}$ SAM and interpret the simulation results as if they were the results of the $t=1$ policy taking place in $t=0$.

2. Calibrate the CGE model to an $\hat{A}^{1}$ SAM updated using one of the solutions to the matrix-balancing problem and interpret the results as if they are close approximations to the true results (those that would be derived if the true $A^{1}$ were known).

Our proposal is to examine the closeness of the simulation results obtained using option 1 ("trueness" of data preferable over "timeliness") with those obtained using option 2 ("timeliness" of data preferable to "trueness") under the three different updating strategies. For this we calibrate the regional CGE model to three alternate SAMs derived using the procedures outlined above. The comparison may help us in appraising the extent to which SAM selection may influence the simulation results and, alternatively, which updating procedure may offer a more reliable, in some sense of proximity, set of simulation results. In our examination, we consider three tax policy scenarios with substantial allocative effects that would best be captured using general equilibrium analysis: 1) a potential reform of the payroll tax (15 percent reduction of effective tax rates); 2) a potential reform of the value-added tax (30 percent increase of effective rates); 3) an implemented reform of the income tax (about 18 percent decrease in effective rates). The selection of these tax policies can be justified as follows. First, the payroll tax and the value-added tax are in need of harmonization with current European Union rates. Second, the adopted income tax reform took actually place in 1999. Third, the general equilibrium interactions of these three tax figures are substantially different, thus providing a broader spectrum for checking the implications of the alternative updating techniques.

\section{DISCUSSION}

In this section we present two types of comparisons. First, we compare the competing SAMs that are obtained by solving the matrix-balancing problem in terms of proximity measures. We have to restrict ourselves to these proxy, subsidiary indicators since we do not have the true 1999 matrix that would be necessary to compute the induced errors between the alternate updates and the true data. Second, we direct our attention to 
comparing the simulations results that would ensue from adopting each candidate SAM as the numerical backbone of the CGE model.

\subsection{Comparing Inputs to the Model}

The balancing problem takes total regional transactions in the 1995 SAM as the base $A^{0}$ matrix and uses a vector of marginals $X$ for 1999 obtained from the official Regional Income and Product Accounts. Three alternative $\hat{A}^{1}$ SAMs are produced using RAS, cross-entropy, and cosine similarity as minimands of the balancing problem. We shall refer to them, respectively, by SAMRAS, SAMCE, and SAMCOS. Table 1 presents some summary proximity (distance) indicators for both coefficients and transactions between the original 1995 SAM and the three 1999 projections, which, to avoid any implicit numerical bias, are unrelated to the adopted loss functions. Among the included statistics we find the standard percentage error (STPE), Theil's U, and Lahr's (2001) weighted absolute difference (WAD). For these three measures, smaller numbers indicate closer proximity between prior information and projected data. Since they do not have any natural upper bounds, we report them in terms of relative proximity by arbitrarily fixing the lowest one equal to unity. We also consider an unrelated index of similarity used by Le Masné (1990) to compare column coefficients in input-output tables that we extend here to the overall SAM coefficient structure. This index lies between 0 and 1 , with the closer being to 1 , the larger the similarity. Finally, we compute the standard Pearson's correlation coefficient as a measure of linear closeness between SAMs. Unlike the previous measures, these two indices have well-defined bounds and are reported at their actual numerical values.

In the coefficients sub-block of Table 1, we observe that in terms of distance RAS yields poorer proximity indicators than both cross-entropy and cosine similarity under all five ad-hoc distance measures. Cross-entropy and cosine similarity indicators are quite close to each other, but the first one gives smaller proximities in four out the five indicators. When we look at the transactions sub-block, however, the situation reverses. RAS has closer proximities in all cases; cross-entropy and cosine similarity are again somewhat close to each other, and each updating comes ahead in two out of the four indicators. These results seem to give support to the assertion by Robinson, Cattaneo, and El-Said (2001) that RAS gives closer approximations than cross entropy in projecting transactions matrices, whereas the opposite occurs in the updating of coefficient matrices. On the other hand, these indicators suggest that cosine similarity gives rise to a more middle-of-the-road compromise solution.

The good news from the data in Table 1 is that there is a clear categorization among the three alternate SAMs in terms of their coefficient or flow proximities to the base 
Table 1

Proximity Measures to SAM95

\begin{tabular}{lccc}
\hline & SAMRAS & SAMCE & SAMCOS \\
\hline COEFFICIENTS & & & \\
STPE & 1.3571 & $\mathbf{1}$ & 1.0931 \\
U (Theil) & 1.2274 & $\mathbf{1}$ & 1.0242 \\
WAD & 1.3516 & $\mathbf{1}$ & 1.1955 \\
Le Masné & 0.8563 & $\mathbf{0 . 8 9 4 1}$ & 0.8843 \\
Pearson & 0.9606 & 0.9732 & $\mathbf{0 . 9 7 3 7}$ \\
TRANSACTIONS & & & \\
STPE & $\mathbf{1}$ & 1.0336 & 1.0869 \\
U (Theil) & $\mathbf{1}$ & 1.0936 & 1.0900 \\
WAD & $\mathbf{1}$ & 1.0448 & 1.1664 \\
Pearson & $\mathbf{0 . 9 2 9 7}$ & 0.9145 & 0.9211 \\
\hline
\end{tabular}

SAM $A^{0}$. On the other hand, there is no conclusive recommendation as to which SAM should be selected in implementing the CGE model. On the one hand, input-output coefficients play a crucial role in the price and commodity equilibrium conditions, which may suggest a preference for using SAMCE; on the other hand, main results are commonly reported in aggregate transaction terms and this perhaps hints at SAMRAS as preferable. Perhaps we could compromise on using SAMCOS since there is no way of knowing a priori the appropriate mix between coefficient and transaction preferability in any given model implementation. Therefore an additional check for further evidence is called for.

\subsection{Comparing Outputs From the Model}

Each SAM is a candidate database for the calibration of the CGE model and its associated benchmark equilibrium. For each equilibrium, we calculate GDP and its decomposition into standard income and expenditure sides both in absolute and percentage levels as well as the government accounts. Table 2 summarizes the results. Unlike the base equilibrium for 1995, which is expressed in current prices, all projected benchmark values are expressed in terms of the selected numeraire (the wage rate).

The benchmark results are seen to be strikingly robust to SAM selection. Even considering that CGE models are not prediction models and that aggregation works to smooth out differences, the degree of similarity among the results generated by adopting the three alternate databases is noteworthy and reassuring as far as performing policy simulations is concerned. This is particularly remarkable when we look at the composition of government tax revenues, a crucial check for the credibility of the simulation results that will be derived from a tax policy model. 
Table 2

Aggregate Indicators for Base SAM 95 and Projected SAM 99 Benchmarks.

\begin{tabular}{|c|c|c|c|c|c|c|c|c|}
\hline & SAM95 & \%GDP & SAMRAS & \%GDP & SAMCE & \%GDP & SAMCOS & \%GDP \\
\hline \multicolumn{9}{|c|}{ MACRO INDICATORS } \\
\hline Wages and salaries & $3,190,651$ & 0.3480 & $4,043,008$ & 0.3354 & $4,043,008$ & 0.3356 & $4,043,008$ & 0.3364 \\
\hline Business income & $4,684,521$ & 0.5109 & $5,965,350$ & 0.4948 & $5,965,349$ & 0.4951 & $5,965,350$ & 0.4964 \\
\hline Net indirect taxes & $1,293,851$ & 0.1411 & $2,047,475$ & 0.1698 & $2,039,981$ & 0.1693 & $2,009,248$ & 0.1672 \\
\hline GDP-Income & $9,169,023$ & 1.0000 & $12,055,833$ & 1.0000 & $12,048,338$ & 1.0000 & $12,017,606$ & 1.0000 \\
\hline Private consumption & $6,276,539$ & 0.6845 & $7,892,806$ & 0.6547 & 7,938,697 & 0.6589 & $7,639,948$ & 0.6357 \\
\hline Investment & $2,554,606$ & 0.2786 & $4,094,765$ & 0.3397 & $4,094,767$ & 0.3399 & $4,094,765$ & 0.3407 \\
\hline Public consumption & $2,001,000$ & 0.2182 & $2,765,039$ & 0.2294 & $2,731,769$ & 0.2267 & $2,730,950$ & 0.2272 \\
\hline Trade balance & $-1,663,122$ & -0.1814 & $-2,696,777$ & -0.2237 & $-2,716,894$ & -0.2255 & $-2,448,057$ & -0.2037 \\
\hline GDP-Expenditure & $9,169,023$ & 0.9999 & $12,055,833$ & 1.0001 & $12,048,339$ & 1.0000 & $12,017,606$ & 0.9999 \\
\hline \multicolumn{9}{|l|}{ GOVERNMENT } \\
\hline Net Production Taxes & $-422,658$ & -0.0461 & $-383,093$ & -0.0318 & $-390,587$ & -0.0324 & $-421,320$ & -0.0351 \\
\hline VAT & 597,476 & 0.0652 & 897,807 & 0.0745 & 897,808 & 0.0745 & 897,807 & 0.0747 \\
\hline Payroll tax & $1,119,033$ & 0.1220 & $1,532,761$ & 0.1271 & $1,532,761$ & 0.1272 & $1,532,761$ & 0.1275 \\
\hline Income Tax & 933,719 & 0.1018 & $1,232,508$ & 0.1022 & $1,232,508$ & 0.1023 & $1,232,508$ & 0.1026 \\
\hline Public spending & $4,092,415$ & 0.4463 & $5,018,894$ & 0.4163 & $5,011,399$ & 0.4159 & $4,980,667$ & 0.4144 \\
\hline Public Deficit & $-1,864,845$ & -0.2034 & $-1,738,909$ & -0.1442 & $-1,738,908$ & -0.1443 & $-1,738,909$ & -0.1447 \\
\hline
\end{tabular}


Tables 3 A-D, 4 A-D, and 5 A-D describe the simulations of a 15 percent decrease in the payroll tax; a 30 percent increase in VAT rates; and a 17.83 percent reduction in income tax rates. Relative prices, activity levels, macro values, and government and welfare indicators are displayed. The selection of units in each benchmark equilibrium yields prices and activity levels normalized to unity; thus the deviation from the unitary initial value is the percentage change in the variable. For relative prices, any change is in terms of the price of the numeraire. Changes in sectoral activity and the unemployment rate, however, can be interpreted as physical changes. Macro, government, and welfare variables are ratio indicators that do not depend on the chosen numeraire.

The analysis of the simulation results again indicates overall robustness. Table 3A, for instance, shows the biggest price impact in Other services and the smallest in Commercial services in all cases. The ordering of sectors from largest to smallest price impact is essentially the same under all three 1999 databases except for a EnergyCommerce switch with SAMCOS. For activity levels, Table 3B shows that the sectors with the highest and lowest impact are the same (Construction and Non commercial services) in all cases. Ordering sectors according to impact, however, is not as robust as it is with relative prices. The average impact on prices and activities, measured by a Consumers Price Index or an Industrial Activity index, varies little across databases.

The reduced labor costs associated with the tax changes result in about a 2.5 percentage point lower unemployment rate using any of the estimated SAMs, a figure not substantially higher than the 2.2 point reduction that the 1995 calibrated model foresees. A similar conclusion can be drawn for the rest of macro variables as seen in Table 3C, which - with the exception of private consumption and the trade deficit under SAMCOS - are similar. The important welfare variables again show a high degree of robustness. For instance, in Table 3D there would be a welfare improvement (as measured by Hicks' equivalent variation) following the payroll tax reduction policy of about 9.7 points over total tax revenue and of about 2.5 points over GDP.

Table 3A

Relative Prices After a 15 Percent Decrease in Payroll Tax

\begin{tabular}{lcccc}
\hline \multicolumn{1}{c}{ SECTORS } & SAM95 & SAMRAS99 & SAMCE99 & SAMCOS99 \\
\hline Agriculture, cattle, forestry and fishing & 0.9823 & 0.9821 & 0.9819 & 0.9813 \\
Extractives & 0.9827 & 0.9821 & 0.9821 & 0.9814 \\
Energy & 0.9852 & 0.9841 & 0.9846 & 0.9836 \\
Manufactures & 0.9830 & 0.9823 & 0.9822 & 0.9809 \\
Construction & 0.9785 & 0.9752 & 0.9758 & 0.9735 \\
Commerce & 0.9837 & 0.9837 & 0.9839 & 0.9839 \\
Transportation \& communications & 0.9829 & 0.9824 & 0.9824 & 0.9817 \\
Other services & 0.9746 & 0.9728 & 0.9728 & 0.9718 \\
Commercial services & 0.9931 & 0.9933 & 0.9932 & 0.9951 \\
Non commercial services & 0.9771 & 0.9753 & 0.9760 & 0.9750 \\
Consumer Price Index & 0.9837 & 0.9832 & 0.9829 & 0.9835 \\
\hline
\end{tabular}


Table 3B

Activity Levels After a 15 Percent Decrease in Payroll Tax

\begin{tabular}{lcccc}
\hline \multicolumn{1}{c}{ SECTORS } & SAM95 & SAMRAS99 & SAMCE99 & SAMCOS99 \\
\hline Agriculture, cattle, forestry and fishing & 1.0117 & 1.0074 & 1.0074 & 1.0095 \\
Extractives & 1.0151 & 1.0107 & 1.0132 & 1.0127 \\
Energy & 1.0175 & 1.0200 & 1.0203 & 1.0230 \\
Manufactures & 1.0152 & 1.0134 & 1.0119 & 1.0127 \\
Construction & 1.0255 & 1.0225 & 1.0206 & 1.0250 \\
Commerce & 1.0214 & 1.0217 & 1.0211 & 1.0218 \\
Transportation \& communications & 1.0161 & 1.0142 & 1.0147 & 1.0136 \\
Other services & 1.0132 & 1.0180 & 1.0200 & 1.0177 \\
Commercial services & 1.0144 & 1.0145 & 1.0146 & 1.0116 \\
Non commercial services & 1.0009 & 1.0016 & 1.0029 & 1.0030 \\
Industrial Activity Indicator & 1.0160 & 1.0164 & 1.0166 & 1.0163 \\
\hline
\end{tabular}

Table 3C

Macroeconomic Indicators After a 15 Percent Decrease in Payroll Tax

\begin{tabular}{lcccc}
\hline \multicolumn{1}{c}{ INDICATORS } & SAM95 & SAMRAS99 & SAMCE99 & SAMCOS99 \\
\hline Unemployment rate change & 0.0220 & 0.0252 & 0.0257 & 0.0247 \\
Wages and salaries/GDP & 0.3606 & 0.3483 & 0.3486 & 0.3492 \\
Business income/GDP & 0.5126 & 0.4965 & 0.4967 & 0.4982 \\
Net indirect taxes/GDP & 0.1268 & 0.1552 & 0.1547 & 0.1526 \\
Private consumption/GDP & 0.6915 & 0.6624 & 0.6665 & 0.6431 \\
Investment/GDP & 0.2810 & 0.3408 & 0.3404 & 0.3420 \\
Public consumption/GDP & 0.2137 & 0.2244 & 0.2218 & 0.2224 \\
Trade balance/GDP & 0.1863 & -0.2275 & -0.2287 & -0.2075 \\
\hline
\end{tabular}

Table 3D

Government and Welfare Indicators After a 15 Percent Decrease in Payroll Tax

\begin{tabular}{lrrrr}
\hline \multicolumn{1}{c}{ INDICATORS } & SAM95 & SAMRAS99 & SAMCE99 & SAMCOS99 \\
\hline Net Production Taxes/GDP & -0.0463 & -0.0319 & -0.0324 & -0.0351 \\
VAT/GDP & 0.0653 & 0.0746 & 0.0746 & 0.0749 \\
Payroll/GDP & 0.1077 & 0.1124 & 0.1125 & 0.1128 \\
Income Taxes/GDP & 0.1029 & 0.1034 & 0.1035 & 0.1038 \\
Public Deficit/GDP & -0.2091 & -0.1504 & -0.1503 & -0.1509 \\
Welfare change/Tax revenues & 0.1088 & 0.0961 & 0.0973 & 0.0978 \\
Welfare change/GDP & 0.0250 & 0.0248 & 0.0251 & 0.0251 \\
Marginal tax burden & -1.7975 & -1.7203 & -1.7547 & -1.7411 \\
\hline
\end{tabular}


Tables 4 A-D report the same variables for the policy scenario in which a 30 percent tax increase in VAT rates is simulated. Under this tax policy, prices tend to increase relative to the numeraire and activity levels tend to fall. There is no substantial difference among the estimated average impacts on prices and activity levels. Highest and lowest price impacts are the same in all versions (Construction and Non commercial services, respectively). In activity levels, the lowest impact is always detected in Energy. The SAMCOS model identifies Construction as the sector with the most impact , but the other models identify Non commercial services. Macro variables as well as government and welfare indicators show a close degree of resemblance. Interestingly, the marginal welfare change is smaller in absolute value with VAT than with the payroll tax, an indication that there may be some margin for efficiency gains if an appropriately designed, revenue-neutral tax reform is enacted.

The simulated results of the income tax reduction appear in Tables 5 A-D. Because the tax reduction does not create any direct distortion in any of the prices, changes in relative prices are seen to be very homogeneous. All calibrated versions detect that the largest price change affects Commercial services, whereas the smallest effect is systematically detected in Other services. Changes in activity levels are more diverse, but all versions detect the largest impact in Commercial services. Energy and Commerce are tied for the second largest impact, with benchmark SAM and SAMRAS detecting Commerce for the second place and SAMCE and SAMCOS detecting Energy instead. On the other hand, all models coincide in detecting Construction as the sector with the largest decrease in activity. Macro and welfare variables are very similar, especially when we look at the simulation results of the three updated SAMs. The induced marginal tax distortion is smaller for the income tax reduction than for either of the other two simulated policies. Thus the model correctly captures that tax distortions issuing from changes in ad valorem indirect taxes will be larger than those originating in the income tax, even if the latter is not quite lump sum.

Table 4A

Relative Prices After a 30 Percent Increase in VAT

\begin{tabular}{lcccc}
\hline \multicolumn{1}{c}{ SECTORS } & SAM95 & SAMRAS99 & SAMCE99 & SAMCOS99 \\
\hline Agriculture, cattle, forestry and fishing & 1.0039 & 1.0082 & 1.0049 & 1.0014 \\
Extractives & 1.0157 & 1.0178 & 1.0158 & 1.0093 \\
Energy & 1.0188 & 1.0242 & 1.0200 & 1.0181 \\
Manufactures & 1.0193 & 1.0219 & 1.0205 & 1.0106 \\
Construction & 1.0235 & 1.0351 & 1.0363 & 1.0323 \\
Commerce & 1.0005 & 0.9985 & 0.9991 & 1.0006 \\
Transportation \& communications & 1.0205 & 1.0266 & 1.0238 & 1.0208 \\
Other services & 1.0073 & 1.0099 & 1.0118 & 1.0160 \\
Commercial services & 1.0016 & 1.0043 & 1.0040 & 1.0034 \\
Non commercial services & 0.9980 & 0.9957 & 0.9972 & 0.9958 \\
Consumer Price Index & 1.0079 & 1.0082 & 1.0076 & 1.0074 \\
\hline
\end{tabular}


Table 4B

Activity Levels After a 30 Percent Increase in VAT

\begin{tabular}{lcccc}
\hline \multicolumn{1}{c}{ SECTORS } & SAM95 & SAMRAS99 & SAMCE99 & SAMCOS99 \\
\hline Agriculture, cattle, forestry and fishing & 0.9949 & 0.9960 & 0.9968 & 1.0001 \\
Extractives & 0.9937 & 0.9971 & 0.9938 & 0.9897 \\
Energy & 0.9881 & 0.9797 & 0.9787 & 0.9720 \\
Manufactures & 0.9895 & 0.9878 & 0.9930 & 0.9973 \\
Construction & 0.9922 & 0.9880 & 0.9958 & 1.0012 \\
Commerce & 0.9886 & 0.9890 & 0.9899 & 0.9889 \\
Transportation \& communications & 0.9879 & 0.9842 & 0.9846 & 0.9877 \\
Other services & 0.9930 & 0.9893 & 0.9877 & 0.9899 \\
Commercial services & 0.9879 & 0.9835 & 0.9842 & 0.9846 \\
Non commercial services & 0.9997 & 0.9995 & 0.9991 & 0.9991 \\
Industrial Activity Indicator & 0.9909 & 0.9891 & 0.9904 & 0.9916 \\
\hline
\end{tabular}

Table 4C

Macroeconomic Indicators After a 30 Percent Increase in VAT

\begin{tabular}{lrrrc}
\hline \multicolumn{1}{c}{ INDICATORS } & SAM95 & SAMRAS99 & SAMCE99 & SAMCOS99 \\
\hline Unemployment rate change & -0.1040 & -0.0119 & -0.0111 & -0.0107 \\
Wages and salaries/GDP & 0.3421 & 0.3289 & 0.3291 & 0.3302 \\
Business income/GDP & 0.4997 & 0.4825 & 0.4828 & 0.4837 \\
Net indirect taxes/GDP & 0.1582 & 0.1886 & 0.1881 & 0.1860 \\
Private consumption/GDP & 0.6749 & 0.6435 & 0.6474 & 0.6246 \\
Investment/GDP & 0.2820 & 0.3442 & 0.3468 & 0.3488 \\
Public consumption/GDP & 0.2188 & 0.2293 & 0.2268 & 0.2276 \\
Trade balance/GDP & -0.1757 & -0.2170 & -0.2210 & -0.2010 \\
\hline
\end{tabular}

Table 4D

Government and Welfare Indicators After a 30 Percent Increase in VAT

\begin{tabular}{lcccc}
\hline \multicolumn{1}{c}{ INDICATORS } & SAM95 & SAMRAS99 & \multicolumn{1}{c}{ SAMCE99 } & SAMCOS99 \\
\hline Net Production Taxes/GDP & -0.0459 & -0.0314 & -0.0322 & -0.0349 \\
VAT/GDP & 0.0841 & 0.0953 & 0.0956 & 0.0957 \\
Payroll/GDP & 0.1199 & 0.1247 & 0.1247 & 0.1252 \\
Income Taxes/GDP & 0.1004 & 0.1005 & 0.1005 & 0.1008 \\
Public Deficit/GDP & -0.1899 & -0.1282 & -0.1281 & -0.1287 \\
Welfare change/Tax revenues & -0.0934 & -0.0885 & -0.0848 & -0.0817 \\
Welfare change/GDP & -0.0242 & -0.0256 & -0.0245 & -0.0234 \\
Marginal tax burden & -1.5058 & -1.4282 & -1.3371 & -1.2827 \\
\hline
\end{tabular}


Table 5A

Relative Prices After a 17.21 Percent Decrease in Income Tax

\begin{tabular}{lcccc}
\hline \multicolumn{1}{c}{ SECTORS } & SAM95 & SAMRAS99 & SAMCE99 & SAMCOS99 \\
\hline Agriculture, cattle, forestry and fishing & 1.0014 & 1.0020 & 1.0018 & 1.0022 \\
Extractives & 1.0013 & 1.0019 & 1.0017 & 1.0020 \\
Energy & 1.0014 & 1.0020 & 1.0018 & 1.0021 \\
Manufactures & 1.0013 & 1.0019 & 1.0017 & 1.0020 \\
Construction & 1.0010 & 1.0014 & 1.0013 & 1.0016 \\
Commerce & 1.0014 & 1.0020 & 1.0018 & 1.0023 \\
Transportation \& communications & 1.0013 & 1.0019 & 1.0017 & 1.0020 \\
Other services & 1.0060 & 1.0009 & 1.0009 & 1.0010 \\
Commercial services & 1.0017 & 1.0025 & 1.0022 & 1.0029 \\
Non commercial services & 1.0007 & 1.0010 & 1.0009 & 1.0010 \\
Consumer Price Index & 1.0013 & 1.0019 & 1.0017 & 1.0021 \\
\hline
\end{tabular}

Table 5B

Activity Levels After a 17.21 Percent Decrease in Income Tax

\begin{tabular}{lcccc}
\hline \multicolumn{1}{c}{ SECTORS } & SAM95 & SAMRAS99 & SAMCE99 & SAMCOS99 \\
\hline Agriculture, cattle, forestry and fishing & 0.9969 & 0.9984 & 0.9985 & 0.9872 \\
Extractives & 0.9928 & 0.9919 & 0.9940 & 1.0017 \\
Energy & 1.0037 & 1.0093 & 1.0112 & 1.0155 \\
Manufactures & 0.9935 & 0.9930 & 0.9879 & 0.9852 \\
Construction & 0.9499 & 0.9544 & 0.9489 & 0.9488 \\
Commerce & 1.0103 & 1.0109 & 1.0097 & 1.0088 \\
Transportation \& communications & 1.0016 & 1.0034 & 1.0041 & 1.0004 \\
Other services & 1.0026 & 1.0037 & 1.0059 & 0.9992 \\
Commercial services & 1.0118 & 1.0147 & 1.0153 & 1.0150 \\
Non commercial services & 1.0005 & 1.0009 & 1.0016 & 1.0016 \\
Industrial Activity Indicator & 0.9987 & 0.9980 & 0.9982 & 0.9976 \\
\hline
\end{tabular}

Table 5C

Macroeconomic Indicators After a 17.21 Percent Decrease in Income Tax

\begin{tabular}{lcccc}
\hline \multicolumn{1}{c}{ INDICATORS } & SAM95 & SAMRAS99 & SAMCE99 & SAMCOS99 \\
\hline Unemployment rate change & -0.0017 & -0.0027 & -0.0024 & -0.0031 \\
Wages and salaries/GDP & 0.3471 & 0.3345 & 0.3348 & 0.3353 \\
Business income/GDP & 0.5121 & 0.4969 & 0.4969 & 0.4985 \\
Net indirect taxes/GDP & 0.1408 & 0.1687 & 0.1683 & 0.1662 \\
Private consumption/GDP & 0.6971 & 0.6682 & 0.6724 & 0.6488 \\
Investment/GDP & 0.2634 & 0.3243 & 0.3224 & 0.3216 \\
Public consumption/GDP & 0.2184 & 0.2298 & 0.2272 & 0.2277 \\
Trade balance/GDP & -0.1789 & -0.2224 & -0.2200 & -0.1981 \\
\hline
\end{tabular}


Table 5D

Government and Welfare Indicators After a 17.21 Percent Decrease in Income Tax

\begin{tabular}{lcrrr}
\hline \multicolumn{1}{c}{ INDICATORS } & \multicolumn{1}{c}{ SAM95 } & \multicolumn{1}{c}{ SAMRAS99 } & SAMCE99 & SAMCOS99 \\
\hline Net Production Taxes/GDP & -0.0456 & -0.0317 & -0.0321 & -0.0347 \\
VAT/GDP & 0.0647 & 0.0737 & 0.0736 & 0.0740 \\
Payroll/GDP & 0.1217 & 0.1267 & 0.1268 & 0.1268 \\
Income Taxes/GDP & 0.0844 & 0.0848 & 0.0848 & 0.0850 \\
Public Deficit/GDP & -0.2217 & -0.1639 & -0.1638 & -0.1642 \\
Welfare change/Tax revenues & 0.0743 & 0.0651 & 0.0658 & 0.0654 \\
Welfare change/GDP & 0.0167 & 0.0165 & 0.0166 & 0.0164 \\
Marginal tax burden & -0.9362 & -0.8710 & -0.8847 & -0.8739 \\
\hline
\end{tabular}

The data displayed in Tables 3 - 5 tentatively suggest that there is a high level of robustness in the simulation outcomes in spite of the intrinsically different nature of the SAM updating procedures. The key variables in any general equilibrium model, however, are prices and quantities. Once we know them, all the other variables and indicators can be easily calculated. Hence, as a final check, we compare prices and activity levels to obtain measures of goodness of fit between the benchmark equilibrium for 1995 and the alternate equilibria calibrated from the three 1999 projected SAMs.

The first descriptive statistic we use is the Pearson correlation coefficient, an index that captures the relative direction and relative magnitudes of the 1999 "predicted" effects vis a vis the 1995 results. The second statistic is a weighted correlation coefficient (see Kehoe, Polo, and Sancho 1995) that captures deviations between the predictions obtained calibrating the model with the base SAM $A^{0}$ and each of the three competing SAMs $\hat{A}^{1}$. In the relative price comparison the weights correspond to the CPI weights, whereas for activity levels we have used value-added shares.

The results are presented in Table 6. An examination of this table shows that all correlations are quite high and the model implemented with the SAM updated with RAS yields, in general, the closest match to 1995 results. Cross entropy and cosine similarity obtain larger correlations only in a very few categories each. Cross entropy comes in second position when we look at prices; when we look at activity levels, cross entropy and cosine similarity alternate shift positions depending on the tax simulation. As for categories, relative prices seem to have a slightly higher prediction power than quantities when we look at indirect tax changes, but the situation reverses when we examine the effects of the income tax reduction. The general outlook is that, as far as correlation measures are concerned, RAS seems to yield larger values but with relatively small quantitative differences. 
Table 6

Relative Prices and Activity Levels Correlations

\begin{tabular}{lccc}
\hline & SAMRAS & SAMCE & SAMCOS \\
\hline $\begin{array}{l}\text { Prices: 15\% payroll tax decrease } \\
\quad \text { Pearson }\end{array}$ & 0.9915 & $\mathbf{0 . 9 9 4 3}$ & 0.9858 \\
$\quad$ Weighted correlation & $\mathbf{0 . 9 9 3 1}$ & 0.9869 & 0.9683 \\
$\begin{array}{l}\text { Prices: 30\% VAT increase } \\
\quad \text { Pearson }\end{array}$ & $\mathbf{0 . 9 7 9 9}$ & 0.9612 & 0.8632 \\
$\quad$ Weighted correlation & $\mathbf{0 . 9 9 1 0}$ & 0.9895 & 0.8784 \\
$\quad \begin{array}{l}\text { Prices: } 17.21 \% \text { income tax decrease } \\
\quad \text { Pearson }\end{array}$ & 0.9982 & 0.9971 & 0.9868 \\
$\quad$ Weighted correlation & 0.9942 & 0.9973 & 0.9773 \\
Activities: $15 \%$ payroll tax decrease & & & \\
$\quad$ Pearson & $\mathbf{0 . 8 9 7 1}$ & 0.8385 & 0.8919 \\
$\quad$ Weighted correlation & 0.9896 & 0.9786 & $\mathbf{0 . 9 8 9 8}$ \\
Activities: 30 \% VAT increase & & & \\
$\quad$ Pearson & & & \\
$\quad$ Weighted correlation & $\mathbf{0 . 8 8 8 9}$ & 0.7885 & 0.6195 \\
Activities: $17.21 \%$ income tax decrease & $\mathbf{0 . 9 8 2 5}$ & 0.9484 & 0.9055 \\
$\quad$ Pearson & & & \\
$\quad$ Weighted correlation & $\mathbf{0 . 9 9 3 0}$ & 0.9859 & 0.9392 \\
$\quad$ & $\mathbf{0 . 9 9 3 6}$ & 0.9823 & 0.9591 \\
\hline
\end{tabular}

\section{CONCLUDING REMARKS}

We reported in this paper a limited empirical test of the performance of three different means of solving the matrix-balancing problem in an economic setting. Instead of focusing exclusively on comparing the resulting updated matrices, we also examined the implications of adopting each competing SAM in the numerical implementation of a regional CGE model. The overall impression is that simulation results are not critically sensitive to the choice of updated database and not very different in turn to results in the base year. This may be partly due to the presence of a rather stable technological and preference environment (Afrasiabi and Casler 1991), but this assumption cannot be checked out without knowing the true SAM.

A second conclusion is that an ex-ante examination of distance measures among alternative SAMs is not necessarily an indication of the ex-post performance of simulations. Recall that RAS gave smaller distance indicators when looking at transactions while cross entropy came ahead when looking at coefficient matrices, with cosine similarity being second on both counts. However, when we evaluate simulation results using correlation coefficients defined over the core equilibrium values of prices and quantities, the indicators hint at RAS as possibly providing a larger predictive power. 
The experiment reported here is a one-shot experiment, and no extrapolations to general conclusions should be drawn at this stage. Further research is clearly required; a possibility for a more systematic testing is using Monte Carlo simulations where instead of an actual marginal vector, a collection of randomly selected ones could be used. Average and dispersion statistics could then be computed to obtain a more balanced view of performance that might be useful in unveiling hidden trends specific to each of the updating strategies. Another consideration is the influence of the structure of the model in terms of closure rules and functional forms. We did not explore these issues here. As a piece of positive evidence, however, Kehoe, Polo, and Sancho (1995) find in a related CGE tax model that simulation results do not appear to be exceedingly sensitive to the selected macroeconomic closure rule. As a piece of negative evidence, however, Adkins, Rickman, and Hameed (2003) show that CGE models with naive Cobb-Douglas cost specifications can yield results substantially different from models where more sophisticated estimates of the cost functions are used.

Finally, more testing and refinements of the information retrieval similarity approach are also necessary. Although vector orthogonality has an interesting economic interpretation, the cosine function does not seem to outperform RAS or cross entropy, coming in second to RAS when transactions matrices matter most and second to cross entropy when coefficients matter most. This middle-of-the road performance, however, could be useful if and when there is no clear-cut modeling preference over the use of transactions or coefficients information.

\section{REFERENCES}

Adkins, L.C., D.S. Rickman, and A. Hameed, 2003. "Bayesian Estimation of Regional Production for CGE Modeling,” Journal of Regional Science 43, 641-661.

Afrasiabi, A. and S.D. Casler, 1991. "Product-Mix and Technological Change Within the Leontief Inverse,” Journal of Regional Science 31, 147-160.

Arndt, C., S. Robinson, and F. Tarp, 2002. "Parameter Estimation for a Computable General Equilibrium Model: A Maximum Entropy Approach,” Economic Modelling 19(3), 375-398.

Bacharach, M., 1970. Biproportional Matrices and Input-output Change. Cambridge University Press: London.

Ballard, C., D. Fullerton, J. Shoven, and J. Whalley, 1985. A General Equilibrium Model for Tax Policy Evaluation. NBER and University of Chicago Press: Chicago.

Cardenete, M.A. and F. Sancho, 2003. "An Applied General Equilibrium Model to Assess the Impact of National Tax Changes on a Regional Economy," Review of Urban and Regional Development Studies 15(1), 55-65.

Golan, A., G. Judge, and S. Robinson, 1994. "Recovering Information from Incomplete or Partial Multisectoral Economic Data," Review of Economics and Statistics 76, 541-549. 
Jackson, R. and T. Murray, 2003. "Alternate Input-Output Matrix Updating Formulations,” Research paper 2003-16, Regional Research Institute, West Virginia University, forthcoming in Economic Systems Research.

Jensen, R., 1980. "The Concept of Accuracy in Regional Input-Output Models," International Regional Science Review 5(2), 139-154.

Jian, X., 2002. "Distance, Degree of Freedom and Error of RAS Method,” working paper presented at the 14th International Conference on Input-Output Techniques, Montreal.

Kehoe, T.J., C. Polo, and F. Sancho, 1995. "An Evaluation of the Performance of an Applied General Equilibrium Model of the Spanish Economy," Economic Theory 6, 115-141.

Kehoe, T. and J. Whalley, 1985. "Uniqueness of Equilibrium in Large Scale Numerical General Equilibrium Models,” Journal of Public Economics 28, 247-254.

Lahr, M., 2001. “A Strategy for Producing Hybrid Regional Input-Output Tables,” in E. Dietzenbacher and M. Lahr (eds.), Input-Output Analysis: Frontiers and Extensions, Palgrave: New York.

Le Masné, P., 1990. "Le Systeme Productif Français face a ses Voisins Europeens,” in E. Archanbault and O. Arkhipof (eds.), La Comptabilité Nationale face au Defí International, Economica: Paris.

McDougall, R., 1999. “Entropy Theory and RAS are Friends,” GTAP working paper No. 6, Purdue University.

Robinson, S., A. Cattaneo, and M. El-Said, 2001. "Updating and Estimating a Social Accounting Matrix Using Cross Entropy Methods,” Economic Systems Research 13(1), 47-64.

Rothblum, U. and H. Schneider, 1989. "Scaling of Matrices Having Preespecified Row Sums and Column Sums," Linear Algebra and its Applications 114/115, 737-764.

Salton, G. and M.J. McGill, 1983. Introduction to Modern Information Retrieval. McGraw-Hill: New York.

Schneider, H., 1989. "Matrix Scaling, Entropy Minimization and Conjugate Duality (I): Positivity Conditions,” Linear Algebra and its Applications 114/115, 785-813.

Schneider, M. and S. Zenios, 1990. "A Comparative Study of Algorithms for Matrix Balancing,” Operations Research 38(3), 439-455.

Shoven, J. and J. Whalley, 1984. "Applied General Equilibrium Models of Taxation and International Trade,” Journal of Economic Literature 22(3), 1007-1051.

Szyrmer, J., 1989. "Trade-Off Between Error and Information in the RAS Procedure,” in R. Miller, K. Polenske, and A. Rose (eds.), Frontiers of Input-Output Analysis, Oxford University Press: Oxford, UK.

Thissen, M. and H. Logfren, 1999. "A New Approach to SAM Updating with an Application to Egypt,” Environment and Planning A 30, 1991-2003. 